\title{
Stereotactic radiation therapy for inoperable, early-stage non-small-cell lung cancer
}

\section{Max Dahele MBChB, Anthony Brade MDCM PhD, Shannon Pearson MRT(T), Andrea Bezjak MDCM}

$\mathrm{T}$ he gold standard treatment for early-stage non-small-cell lung cancer is surgical resection. Yet operative risk is prohibitive for many patients with this condition. Over half of patients who are given a diagnosis of lung cancer are 70 years of age or older. The prevalence of comorbidities in such patients increases with advanced age and a history of smoking. In about $20 \%$ of patients who have early-stage non-small-cell lung cancer (e.g., a single tumour with no evidence of regional lymphadenopathy or distant metastases), the cancer is considered "medically inoperable" because the patient is deemed unable to undergo surgery.

The historical standard treatment for inoperable non-smallcell lung cancer in such patients has been radical radiotherapy. Typically, a dose of $60 \mathrm{~Gy}$ or greater is delivered in daily 2-Gy fractions over 6 weeks. Three-dimensional conformal techniques, which shape the radiation beam to the tumour and surrounding area, are usually used. But radical radiotherapy has been found to achieve long-term, local control of tumours in only $30 \%$ of patients, ${ }^{2}$ a $2-3$-fold lower proportion than patients whose tumours are treated surgically. ${ }^{3}$ A more effective alternative is needed.

Stereotactic body radiation therapy, known generally as SBRT, is an emerging treatment that should be considered for early-stage, peripheral non-small-cell lung cancer in patients who cannot undergo surgery, including frail patients with severe pulmonary comorbidities.

\section{What is stereotactic radiation therapy?}

Stereotactic radiation therapy exploits advances in technology to deliver multiple beams of high-dose, highly conformal radiation in short courses. The doses per fraction that it delivers are far higher than those of conventional radiotherapy (e.g., 12-20 Gy v. 2-3 Gy per fraction in conventional radiotherapy) and its overall period of treatment is much shorter (e.g., 1-2 weeks rather than 5-6 weeks). These characteristics make stereotactic radiotherapy much more potent (i.e., radioablative) than conventional radiotherapy for treating cancerous tissues, but potentially much more damaging to normal tissues. Highly precise and accurate delivery of radiation is therefore a necessity. Since the first use of extracranial stereotactic radiotherapy in 1991, groups in Europe, Japan and North America have refined its use for the

\section{Key points}

- Stereotactic body radiation therapy (SBRT) is an alternative to conventional radiotherapy for patients unfit for surgery who have early-stage, peripheral non-small-cell lung cancer.

- Compared with conventional radiotherapy, stereotactic radiotherapy delivers doses of radiation that are much more potent, resulting in higher rates of local control.

- Stereotactic radiotherapy is generally well tolerated, even in patients who are frail or elderly or have comorbid cardiopulmonary disease.

- Early symptoms of toxicity (e.g., cough, low-grade fatigue) are usually mild, and rates of late symptoms (e.g., pain in the chest wall, fractures of the ribs) are low. Deaths and other serious adverse events are uncommon.

treatment of lung cancer. Different treatment regimens and methods have emerged.

\section{How is it delivered for lung cancer?}

Treatment of pulmonary cancer with stereotactic radiotherapy can be given on an outpatient basis. Fractions of radiation are often administered a few days apart rather than on successive days. Typical doses are 12-20 Gy per fraction, for a total of 45-60 Gy in 3-5 fractions. The tumour being treated must be identified precisely and reliably throughout the 30-60 minute treatment session. Millimetre-level accuracy can be achieved with the addition of high-quality imaging tools, such as a cone-beam computed tomography (CBCT) unit mounted on the radiation treatment machine (Figure 1). With the tumour made visible through CBCT scanning, the position of the patient can be guided using exact coordinates and the field of treatment can be centred more precisely on the tumour. Less of a margin around the tumour is then needed to account for day-to-day variations in positioning. The result is a reduction in the volume of normal tissue that is irradiated and a consequent reduction in toxicity.

From the Radiation Medicine Program (Dahele, Brade, Pearson, Bezjak), Princess Margaret Hospital, University Health Network; and the Department of Radiation Oncology (Dahele, Brade, Bezjak), University of Toronto, Toronto, Ont. 

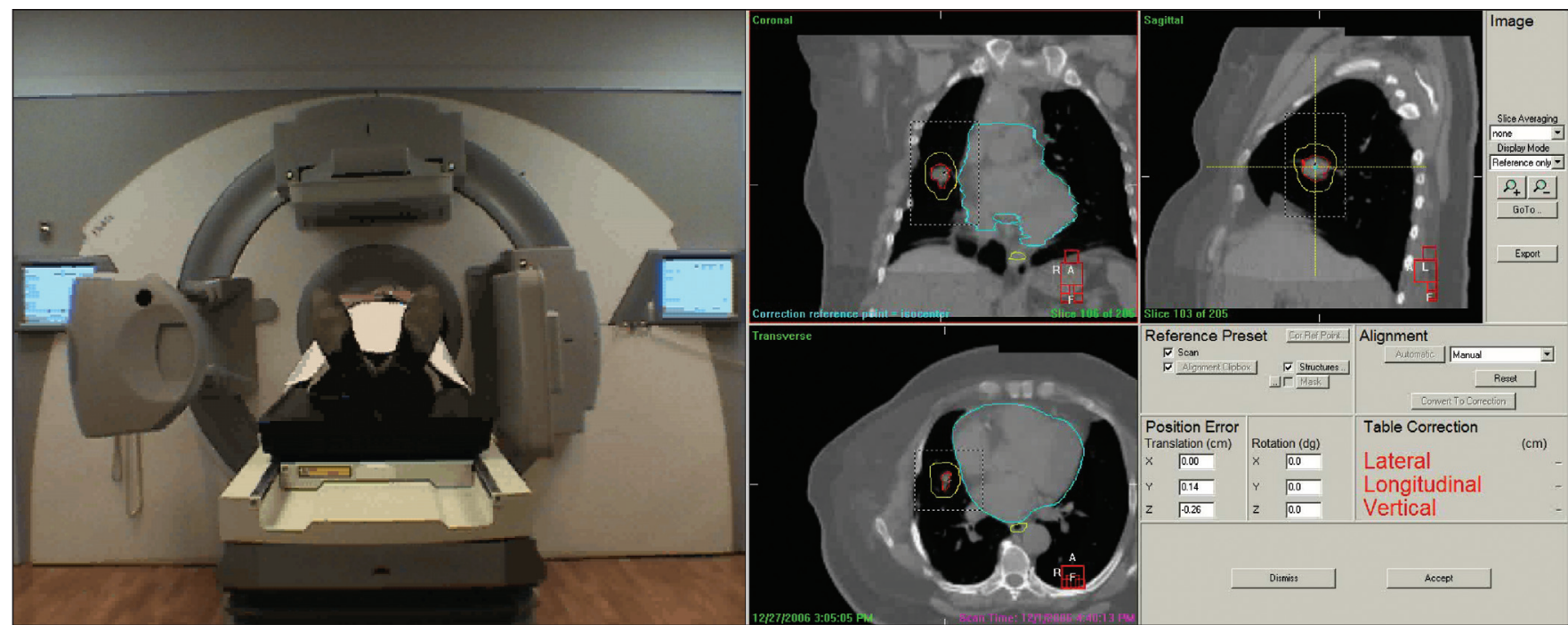

Figure 1: A radiation treatment machine (linear accelerator), left. The extended side arms to the left and right of the treatment couch make up the on-board cone-beam computed tomography (CBCT) scanner. Right, 3 CBCT scans show the location of a tumour and normal tissues in 3-dimensional form, enabling the targeted region (i.e., the tumour) to be positioned with millimetre-accuracy before and during the treatment session.

Further reductions in the irradiation of normal tissue can be achieved by using imaging before treatment to evaluate the motion of the tumour as it moves with the breathing movements of the patient. This allows the region of high-dose radiation to be tightly conformed to the motion. In recognition of the potency of the doses used in stereotactic body radiotherapy, no margin is added to the gross tumour that is visible on pretreatment planning CT scans. In conventional radiotherapy, such a margin is added to allow for invisible, microscopic extension. To limit and shape the high-dose volume of radiation delivered during a treatment session, stereotactic radiotherapy employs many more beams of radiation than are used in conventional, 3dimensional conformal radiotherapy (e.g., 7-13 beams rather than 3-4). Each beam contributes a small portion of the total dose, with the aim of reducing damage along its path (Figure 2).

\section{Who is eligible?}

Experience to date indicates that stereotactic radiotherapy for lung cancer is effective and well tolerated, ${ }^{4-6}$ even by frail patients with severely compromised lung function. Not all patients with inoperable non-small-cell lung cancer are eligible for stereotactic body radiotherapy. The location of a tumour is an important consideration. Tumours that are in close proximity to central structures (e.g., trachea, main bronchi) are generally excluded because of the prohibitive risk of serious toxicity (potentially fatal) if large doses are delivered to critical structures. ${ }^{7}$ Therapy may also be contraindicated in patients with tumours that are in close proximity to the spinal cord, aorta or brachial plexus. Patients must be able to maintain the treatment position for the entire treatment session (frequently 30-60 minutes versus
10-15 minutes for conventional radiotherapy). The administration of analgesia (e.g., if there is shoulder pain associated with elevating the arms above the head) or an anxiolytic may be necessary.

\section{What are the possible harms?}

In patients with peripheral tumours, stereotactic radiotherapy is typically well tolerated. The incidence of serious toxicity is typically $5 \%$ or lower. A recent study found that stereotactic radiotherapy did not cause adverse effects on quality of life in the first year after treatment. ${ }^{8}$ The most common early symptoms of toxicity (i.e., occurring 3 months or less after stereotactic body radiotherapy) include low-grade fatigue, cough, erythema and pain in the chest wall. Rates of late symptoms of toxicity (i.e., occurring more than 3 months after poststereotactic body radiotherapy) have been low. Late occurrences of rib fractures and chest wall pain have been observed in some patients with peripheral tumours in whom part of the chest wall often receives the full dose of radiation. Reports of treatment-related mortality (e.g., radiation pneumonitis, bleeding and esophageal ulceration) have been rare, although constant vigilance is necessary. ${ }^{9}$ Many clinical series have been unable to determine the true risk-benefit ratio of stereotactic radiotherapeutic regimens for lung cancer because of relatively short follow-up periods, a low event rate and competing risk of death.

\section{What is the evidence so far?}

The literature on stereotactic body radiotherapy for lung cancer currently consists of single or multi-institutional studies of patients treated using stereotactic body radiotherapy protocols, with some dose-escalation series but no randomized 


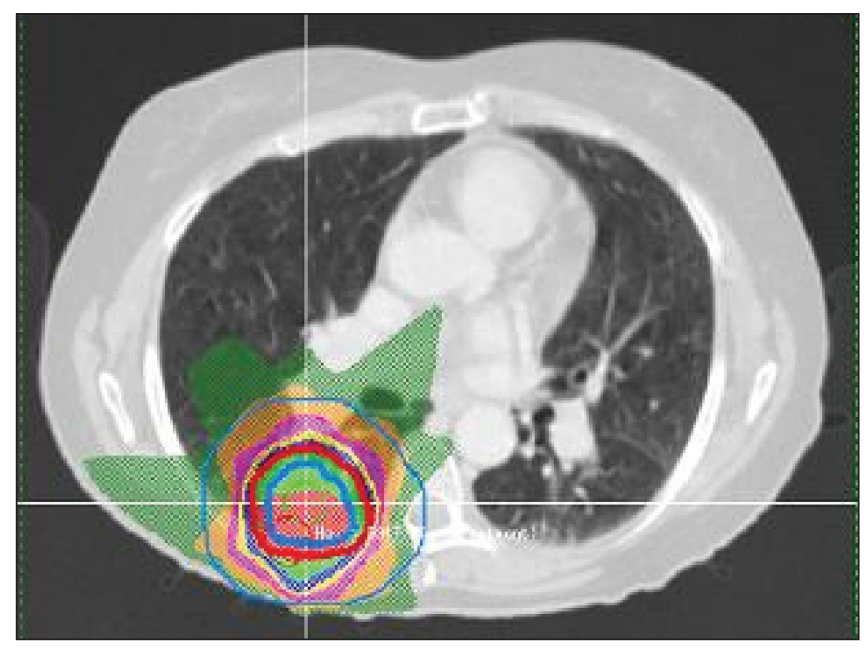

Figure 2: Computed tomography scan of the chest showing regions of radiation that conform to a tumour (green $=18 \mathrm{~Gy}$, orange $=30 \mathrm{~Gy}$, yellow $=54 \mathrm{~Gy}$, blue $=60 \mathrm{~Gy}$, red $=80 \mathrm{~Gy}$ ). Multiple beams of radiation converge from a wide range of angles and planes.

studies yet. Rates of local control of tumours are typically $80 \%-90 \%,{ }^{4,5,7}$ which represents a $2-3$-fold improvement over rates for conventionally fractionated radiotherapy. Operable patients who declined surgery and received stereotactic body radiotherapy had an actuarial 5-year overall survival rate of $70 \%$. This rate is comparable to that for surgical resection, ${ }^{4}$ and suggests that stereotactic body radiotherapy may be a safe alternative to surgery. Randomized trials are underway to compare stereotactic body radiotherapy to surgery. Some authors have suggested that stereotactic body radiotherapy should represent a new standard of care, ${ }^{4}$ whereas others contend that more study is needed. ${ }^{9}$ Given the potential for late adverse effects of radiotherapy, continued registration of patients in prospective trials or institutional protocols is needed.

\section{What can we expect in the future?}

Studies that are currently underway are addressing some of the limitations in the current evidence base supporting stereotactic body radiotherapy. Early comparisons suggest that use of stereotactic radiotherapy may achieve a rate of local control of tumours double that obtained with conventional radiotherapy. This possibility is now being evaluated by prospective, randomized phase II studies. Other prospective studies include phase II and III studies comparing stereotactic body radiotherapy and surgery in operable, early-stage non-small-cell lung cancer and a phase I-II dose-escalation study for patients with central tumours. Long-term data from such studies will help to document the incidence and significance of late radiation effects. Institutional programs must emphasize adequate training, rigorous quality assurance and robust clinical process to emulate the results obtained in trials. ${ }^{6}$

This article has been peer reviewed.

Competing interests: None declared.

Funding: Research funding for the Stereotactic Lung Radiotherapy Program at Princess Margaret Hospital is provided by the Elekta Synergy Research Group, Elekta Ltd., Crawley, UK; and the Addie MacNaughton Chair in Lung Cancer Radiotherapy, Princess Margaret Hospital, Toronto, Ont.

\section{REFERENCES}

1. Raz DJ, Zell JA, Ou SH, et al. Natural history of stage I non-small cell lung cancer: implications for early detection. Chest 2007;132:193-9.

2. Rowell NP, Williams CJ. Radical radiotherapy for stage I/II non-small cell lung cancer in patients not sufficiently fit for or declining surgery (medically inoperable): a systematic review. Thorax 2001;56:628-38.

3. El-Sherif A, Gooding WE, Santos R, et al. Outcomes of sublobar resection versus lobectomy for stage I non-small cell lung cancer: a 13-year analysis. Ann Thorac Surg 2006;82:408-15, discussion 415-6.

4. Onishi H, Shirato H, Nagata Y, et al. Hypofractionated stereotactic radiotherapy (HypoFXSRT) for stage I non-small cell lung cancer: updated results of 257 patients in a Japanese multi-institutional study. J Thorac Oncol 2007;2(Suppl 3):S94-100.

5. Lagerwaard FJ, Haasbeek CJ, Smit EF, et al. Outcomes of risk-adapted fractionated stereotactic radiotherapy for stage I non-small-cell lung cancer. Int J Radiat Oncol Biol Phys 2008;70:685-92.

6. Dahele M, Pearson S, Purdie T, et al. Practical considerations arising from the implementation of lung stereotactic body radiation therapy (SBRT) at a comprehensive cancer center. J Thorac Oncol 2008;3:1332-41.

7. Timmerman R, McGarry R, Yiannoutsos C, et al. Excessive toxicity when treating central tumors in a phase II study of stereotactic body radiation therapy for medically inoperable early-stage lung cancer. J Clin Oncol 2006;24:4833-9.

8. Lagerwaard FJ, van der Geld Y, Slotman BJ, et al. Quality of life after stereotactic radiotherapy for medically inoperable stage I lung cancer [abstract]. Int J Radiat Oncol Biol Phys 2006;66(Suppl 1):S133-4.

9. Ball D. Stereotactic radiotherapy for non small cell lung cancer. Curr Opin Pulm Med 2008;14:297-302.

Correspondence to: Dr. Andrea Bezjak, Princess Margaret

Hospital, 610 University Ave., Toronto ON M5G 2M9;

fax416 946-6561; Andrea.Bezjak@rmp.uhn.on.ca

CMAJ invites contributions to Innovations, which highlights recent diagnostic and therapeutic advances. Novel uses of older treatments will also be considered. For publication, the benefits of the innovation, its availability and its limitations must be highlighted clearly, but briefly. Visual elements (images) are essential. Submit brief evidence-based articles (maximum 1000 words and 5 references) to http://mc.manuscriptcentral.com/cmaj or email diane.kelsall@cmaj.ca to discuss ideas. 